\title{
Health Literacy and Pre-Eclampsia Knowledge of Pregnant Mother in Primary Health Care in Surabaya
}

\author{
Daryan Alif Putra ${ }^{1}$, Fundhy Sinar Ikrar Prihatanto ${ }^{2}$, Pudji Lestari $^{3 *}$ \\ ${ }^{1}$ Faculty of Medicine Universitas Airlangga, Surabaya, Indonesia \\ ${ }^{2}$ Department of Anatomy and Histology, Faculty of Medicine, Universitas Airlangga Surabaya, Indonesia \\ ${ }^{3}$ Department of Public Health and Preventive Medicine, Faculty of Medicine, Universitas Airlangga Surabaya, Indonesia
}

A R T I C L E I N F O

\section{Article history:}

Received 14 September 2020

Received in revised form 27

October 2020

Accepted 29 October 2020

Available online 31 October 2020

\section{Keywords:}

Preeclampsia,

Health Literacy,

Pregnancy,

Antenatal Care.

*) Corresponding author:

pulesaja@gmail.com

\begin{abstract}
A B S T R A C T
Introduction: Health literacy is defined as individual's ability to gather, communicate, process, and understand basic health information and services to make appropriate health decision. Preeclampsia is a hypertensive disorder that occur during pregnancy and proteinuria at least $0.3 \mathrm{~g}$ in 24 hour urine collection. The purpose of this study was to find the connection between health literacy and knowledge of pre-eclampsia in Surabaya.

Methods: A cross sectional study of second and third semester pregnant mother in Mojo and Pucang Sewu primary health care area in Surabaya. The study used modified SAHL-E test and modified questionnaire for knowledge of pre-eclampsia. Data processing used Chi square.

Results: From the total of 79 participant, most attended high school around $47 \%$, primigravida $54 \%$, have occupation of housewife $53 \%$, husband occupation of private employment $87 \%$, majority of participant have good health literacy $67 \%$, and most of the participant have poor knowledge of preeclampsia 59\%. There was a significant relationship between health literacy and the knowledge of pre-eclampsia $(\mathrm{p}=0.029)$.

Conclusion: There was a relationship between health literacy of the participants and their knowledge of Pre-eclampsia. Healthcare provider should have better communication and giving information regarding pregnancy or pre-eclampsia and explain it in a way the patient can understand it well depending on their health literacy.
\end{abstract}

\section{Introduction}

Health literacy is defined as the ability of which an individual able to gather, communicate, process, and understand basic health information and services to make appropriate health decision. ${ }^{1}$ The importance of health literacy especially in women is to make information and services to woman's health, chronic disease, and reproductive health more available, able to enhance woman's communication about their health decision, and take the appropriate actions to implement the decision they make about their health. ${ }^{2}$

Health seeking behaviour is a complex process which has multiple factors that can cause different outcomes. One of the theories that explain health seeking behaviour is using the health belief model. This model is made for the purpose of examining patient response to symptoms and compliance to health treatment. Health belief model constructs six main pillars for health behaviour which are: perceived susceptibility, perceived barriers, perceived benefit, selfefficacy, modifying variables and cues to action. ${ }^{3}$
In 2017, it is estimated that Indonesia have the total of 261 million people with 130 million are females. According to Central Bureau of Statistics Republic of Indonesia, it was estimated around 3.4 million people are still illiterate in Indonesia. The number of mother with high risk in Surabaya during 2015 are around 6,526 and if left untreated, these mothers can have babies with low birth weight, miscarriage and even maternal death. Maternal mortality ratio in Surabaya in 2015 was around 87 per 100,000 living birth. ${ }^{4}$

From the information above there is still in need for research that looked at the connection between health literacy and health seeking behaviour of pre-eclampsia in Surabaya. 
first and second trimester visited Mojo and Pucang Sewu primary health center and the sampling technique used was accidental sampling. The inclusion criteria were mother in their first and second trimester of pregnancy who visited Mojo and Pucang Sewu primary health center and exclusion criteria was pregnant mother who were already at risk to develop preeclampsia.

The research used translated Short Assessment of Health Literacy English (SAHL-E) test for measuring the health literacy5. SAHL-E test is a short question that can measure the level of health literacy from the scale of 0 to 18 and if the score is lower than 14 then the participant have low health literacy. Pre-eclampsia questionnaires were used for finding the subjects knowledge about pre-eclampsia. Other characteristics such as age, level of education, number of children, and occupation of the participant and their husband are also used for this study. The research used chi-square and spearman's rho for statistical test on SPSS and the data analysis were described with central tendency and dispersions as well as using appropriate table and $\mathrm{p}<0.05$ was considered to be significant6. The research had been approved by ethical committee Medical Faculty Airlangga University (239/EC/KEPK/FKUA/2018).

\section{Results}

Of the total 79 subjects, most are at the age of 21-25 years old. The majority of participant graduated from high school around $47 \%$. More than half of participant are primigravida 54\%. Majority occupation for the participant are housewife $53 \%$ while their husband occupation mostly are private employment $87 \%$. Majority of participant have good health literacy $67 \%$ but majority of participants poor knowledge of preeclampsia $59 \%$.

Table.1 Age, Education, Number of children, Occupation, Husband occupation, Health literacy test, and Knowledge of pre-eclampsia subject characteristics

\begin{tabular}{lll}
\hline \multicolumn{1}{c}{ Variable } & $\mathrm{n}=79$ & $\%$ \\
\hline Age & & \\
\hline $21-25$ & 31 & 39 \\
\hline $26-30$ & 23 & 29 \\
\hline Education & & \\
\hline High school & 37 & 47 \\
\hline Bachelor & 30 & 38 \\
\hline Number of Children & & \\
\hline 0 & 43 & 54 \\
\hline 1 & 30 & 38 \\
\hline Mother's Occupation & & \\
\hline Housewife & 42 & 53 \\
\hline Father's Occupation & & \\
\hline Private employment & 69 & 87 \\
\hline Health Literacy test & & \\
\hline Good health literacy & 53 & 67 \\
\hline Poor health literacy & 26 & 33 \\
\hline Knowledge of Pre-eclampsia & & \\
\hline Good knowledge & 37 \\
\hline Poor knowledge & & \\
\hline
\end{tabular}

After determining participant's health literacy, knowledge of pre-eclampsia and health seeking behavior, then it is time to find the association. From table 2, it show that there was a significant relationship between health literacy and knowledge of pre-eclampsia. On the other hand, there was no significant correlation $(p=0.276)$ between health literacy and health seeking behavior.

Table 2. Correlation between health literacy and knowledge of pre-eclampsia.

\begin{tabular}{|c|c|c|c|}
\hline & \multicolumn{2}{|c|}{$\begin{array}{l}\text { Knowledge of } \\
\text { Pre-Eclampsia }\end{array}$} & \multirow[t]{2}{*}{ p value* } \\
\hline & Poor & Good & \\
\hline Poor Health Literacy & $15(19 \%)$ & $11(14 \%)$ & 0.029 \\
\hline Good Health Literacy & $17(22 \%)$ & $36(46 \%)$ & \\
\hline
\end{tabular}

*Chi-square test, $\mathrm{p}<0.05$ considered as significant

Table 3. Correlation between health literacy and health seeking behaviour

\begin{tabular}{lc}
\hline Health Literacy and Health Seeking Behaviour & p value* \\
\hline * Spearman's rho test, $\mathrm{p}<0.05$ considered as significant
\end{tabular}

\section{Discussion}

Based on this study, it shows that the better the health literacy of the mother, then she will have better knowledge of pre-eclampsia and vice versa. A study showed that women with low health literacy can affect their ability to seek healthcare, and adherence to care plans. ${ }^{7}$ While another study showed that women who scored better at question regarding pre-eclampsia are those who have high literacy, multiparty and exposed to information regarding of pre-eclampsia from clinics. ${ }^{8}$ From the two study above, it indicates that women or even health care worker who do not read or informed regarding pre-eclampsia could potentially receive late treatment and subsequently getting complication of pre-eclampsia. Also it can display a theory that the better the literacy of a woman, the more chances she could know information regarding woman's health especially on this case about pre-eclampsia. It is crucial for health care provider to note that it will be easier to informed women with high literacy about health related information rather having low literacy. This also mean that extra care must be used when dealing with women who have lo literacy by using colloquial spoken language, and using a piece of information that easily digest and take home with.

This study shows most of the subjects were at the age of 21-25 years old. Recent study showed that respondent from old age group have more chance to be having low health literacy when compared to younger age group such as those from the age 18 until 34 years old. ${ }^{9}$ The research may not looked into connection between age and health literacy. However, it is important for note that despite low score of knowledge regarding pre-eclampsia, pregnant women at the age of 21-25 years old still have better opportunity to increase their health literacy which then potentially improve their knowledge of pre-eclampsia.

Majority of subjects were high school graduate. A research found that women who have high education tend 
to visit antenatal care more often that woman who have low education. ${ }^{10}$ This show the importance of education to individual's conscience regarding their own health. more than half of the woman (54\%) are on their first pregnancy. A study show that there is no significant correlation between knowledge about health between women who were at their first or second pregnancy. ${ }^{11}$ Based on the study above means that having more experience in pregnancy from having more children, does not guaranteed increase health literacy of a woman for pregnancy. Only more frequent antenatal visit could potentially increase a pregnant woman health literacy.

More than half of participant were housewives. A study show that large number of housewife are having low health literacy, but that is as the result from them having low education. ${ }^{12}$ However, another study show that female who had good income and high education level would likely have a good health literacy. ${ }^{13}$ Both study show that women's health literacy is not directly affected by their occupation, rather to their education level. Being a housewife alone should not be a predictor to a woman's health literacy. Majority of participant's husband are private worker. The husband has the decisive vote on deciding whether his wife could go to get health care due to them being the financial support. ${ }^{14}$ Health care workers should note that having the support from husband could help pregnant woman to seek health care more often. In addition, a person perspective, values, beliefs, and behaviours regarding health and illness varied depending on their culture. As the result of that, health literacy may varied depending on the culture as an example in western country such as America, the people need more westernised biomedical perspective to understand health literacy. ${ }^{15}$ A mother's health literacy can be affected by the culture and thus requiring medical personnel to understand the local culture and use appropriate mean to make the mothers around that culture to understand about health. ${ }^{15}$

A study shows the better health literacy of a mother, then the better knowledge she will have about preeclampsia. Community health workers who have no formal medical training failed to refer pregnant woman who have symptoms of pre-eclampsia even though they help women in the community to labour. ${ }^{16}$ From the study above, it indicates that women or even health care worker who do not read or informed regarding pre-eclampsia could potentially receive late treatment and subsequently getting complication of pre-eclampsia. Also from other studies above, it can display a theory that the better the literacy of a woman, the more chances she could know information regarding woman's health especially on this case about pre-eclampsia. To improve the mother's health literacy, health care provider could use brochures or pamphlet filled by information during pregnancy and pre-eclampsia.

There were some limitations to this study. First, it was only included two primary health centers used and should include all primary health center that have antenatal care. Second, the translated SAHL-E test had not used before on other study. Third, the questionnaires regarding preeclampsia not being used before on other study.

\section{Conclusion}

In conclusion, there was a correlation between health literacy of the participants and their knowledge of preeclampsia. Recommendations for future research are to increase the number of primary health centre included on the research, if possible the whole primary health care in Surabaya is covered, Improve the questions for testing knowledge of pre-eclampsia, and Include other women who are not pregnant who also visit primary health centre to take part answering questionnaire.

\section{Acknowledgements}

The author thank all the staff at Mojo and Pucang Sewu primary health center for assisted data collection, all the participant of the research and to dr. Samsriyaningsih Handayani, M.Kes., M.Ed., Ph.D for guidance writing the research report.

\section{Conflict of Interest}

The author declare there is no conflict of interest

\section{References}

1. Prevention CfDCa. What is Health Literacy? Georgia: Centers for Disease Control and Prevention, 2016.

2. Organization WH. Health Literacy and Health Behaviour. Geneva: World Health Organization, 2009.

3. Nutbeam D. The Evolving Concept of Health Literacy. Social Science \& Medicine. 2008; 67: 2072-8.

4. Timur DKPJ. Profil Kesehatan Surabaya. Surabaya: Dinas Kesehatan Provinsi Jawa Timur, 2015.

5. Quality AfHRa. Health Literacy Measurement Tools. In: Services DoHaH, (ed.). Maryland: Agency for Healthcare Research and Quality, 2018.

6. Corrarino JE. Health Literacy and Women's Health: Challenges and Opportunities. Journal of Midwifery \& Women's Health. 2013; 58: 257-64.

7. You WB, Wolf M, Bailey SC, et al. Factors Associated with Patient Understanding of Preeclampsia. Hypertension in Pregnancy. 2012; 31: 341-9.

8. Protheroe J, Whittle R, Bartlam B, Estacio EV, Clark L and Kurth J. Health Literacy, Associated Lifestyle and Demographic Factors in Adult Population of an English City: A Cross-Sectional Survey. Health Expectations. 2017; 20: 112-9.

9. Muyunda B, Makasa M, Jacobs C, Musonda P and Michelo C. Higher Educational Attainment Associated with Optimal Antenatal Care Visits among Childbearing Women in Zambia. Frontiers in Public Health. 2016; 4.

10. Ohnishi M, Nakamura K and Takano T. Improvement in Maternal Health Literacy among Pregnant Women Who Did Not Complete Compulsory Education: Policy Implications for Community Care Services. Health Policy. 2005; 72: 157-64.

11. Akbarinejad F, Soleymani M and Shahrzadi L. The Relationship between Media Literacy and Health Literacy among Pregnant Women in Health Centers of Isfahan. Journal of Education and Health Promotion. 2017; 6: 17-.

12. Ansari H, Almasi Z, Ansari-Moghaddam A, et al. Health Literacy in Older Adults and Its Related Factors: A Cross-Sectional Study in Southeast Iran. Health Scope. 2016; 5: e37453.

13. C E, Obiora O and Nwankwo C. Health Seeking Behaviour amongst Pregnant Women Attending Antenatal Clinic in Primary Health Care Centres in Rural Communities of Nnewi North L.G.A Anambra State. 2016.

14. Institute of Medicine Committee on Health L. Health Literacy: A Prescription to End Confusion. In: Nielsen-Bohlman L, Panzer AM and Kindig DA, (eds.). Washington (DC): National Academies Press (US) Copyright 2004 by the National Academy of Sciences. All rights reserved., 2004.

15. Boene H, Vidler M, Augusto O, et al. Community Health Worker Knowledge and Management of Pre-Eclampsia in Southern Mozambique. Reproductive Health. 2016; 13: 105. 